\title{
Blood transcriptomics of drug-naïve sporadic Parkinson's disease patients
}

\author{
Raffaella Calligaris ${ }^{1}$, Mihaela Banica ${ }^{2}$, Paola Roncaglia ${ }^{1,8}$, Elisa Robotti ${ }^{3}$, Sara Finaurini ${ }^{1}$, Christina Vlachouli ${ }^{1}$, \\ Lucia Antonutti ${ }^{2}$, Francesco lorio ${ }^{4,8}$, Annamaria Carissimo ${ }^{4}$, Tatiana Cattaruzza ${ }^{2}$, Andrea Ceiner ${ }^{5}$, Dejan Lazarevic ${ }^{1,6}$, \\ Alberto Cucca ${ }^{2}$, Nicola Pangher ${ }^{5}$, Emilio Marengo ${ }^{3}$, Diego di Bernardo ${ }^{4,7}$, Gilberto Pizzolato ${ }^{\wedge}$ and Stefano Gustincich ${ }^{1^{*}}$
}

\begin{abstract}
Background: Parkinson's disease (PD) is a chronic progressive neurodegenerative disorder that is clinically defined in terms of motor symptoms. These are preceded by prodromal non-motor manifestations that prove the systemic nature of the disease. Identifying genes and pathways altered in living patients provide new information on the diagnosis and pathogenesis of sporadic PD.
\end{abstract}

Methods: Changes in gene expression in the blood of 40 sporadic PD patients and 20 healthy controls ("Discovery set") were analyzed by taking advantage of the Affymetrix platform. Patients were at the onset of motor symptoms and before initiating any pharmacological treatment. Data analysis was performed by applying Ranking-Principal Component Analysis, PUMA and Significance Analysis of Microarrays. Functional annotations were assigned using GO, DAVID, GSEA to unveil significant enriched biological processes in the differentially expressed genes. The expressions of selected genes were validated using RT-qPCR and samples from an independent cohort of 12 patients and controls ("Validation set").

Results: Gene expression profiling of blood samples discriminates PD patients from healthy controls and identifies differentially expressed genes in blood. The majority of these are also present in dopaminergic neurons of the Substantia Nigra, the key site of neurodegeneration. Together with neuronal apoptosis, lymphocyte activation and mitochondrial dysfunction, already found in previous analysis of PD blood and post-mortem brains, we unveiled transcriptome changes enriched in biological terms related to epigenetic modifications including chromatin remodeling and methylation. Candidate transcripts as CBX5, TCF3, MAN1C1 and DOCK10 were validated by RT-qPCR.

Conclusions: Our data support the use of blood transcriptomics to study neurodegenerative diseases. It identifies changes in crucial components of chromatin remodeling and methylation machineries as early events in sporadic PD suggesting epigenetics as target for therapeutic intervention.

Keywords: Blood transcriptomics, Parkinson's disease, de novo, Drug-naïve patients

\section{Background}

Parkinson's disease (PD) is a slowly progressive degenerative disorder of the central nervous system (CNS) that is classically defined in terms of motor symptoms consequent to degeneration of specific subsets of mesencephalic dopaminergic (DA) cells within Substantia Nigra (SN) pars compacta. Although DA drugs are effective in alleviating motor symptoms in PD patients, no

\footnotetext{
* Correspondence: gustinci@sissa.it

Deceased

${ }^{1}$ Area of Neuroscience, International School for Advanced Studies (SISSA), via Bonomea 265, 34136 Trieste, Italy

Full list of author information is available at the end of the article
}

pharmacological treatment is currently available to slow or arrest the neurodegenerative process. Furthermore, accurate early diagnosis suffers from the lack of reliable biomarkers. This is due at least in part to three challenges. First, at the onset of the motor symptoms, dopamine depletion in the putamen is $80 \%$, with $60 \%$ of the SN DA neurons already lost [1], proving that at the time of clinical trials neuronal networks are already largely compromised. Moreover, PD is not a homogeneous disease since it presents a plethora of different clinical forms with unclear molecular differences and consequences on the treatment of choice. Finally, the aetiology 
and the initial molecular events of the disease remain unknown since the injured tissue in living patients is not accessible to genomics and biochemical analysis.

PD presents a variety of neuropsychiatric, autonomic, sensory, and sleep disorders that may precede the expression of motor disturbances by more than a decade suggesting that PD is a systemic disease [2]. In this context, a long and intriguing list of alterations of blood physiology has been described in PD patients [3].

Gene expression profiles represent a powerful tool to study the molecular basis of a systemic disease in living patients. Many proof - of - concept studies have been reported to use them as surrogates for disease prediction and classification [4]. Recently, gene expression analysis has identified changes in blood correlated to neurodegeneration in PD [5-11]. However, some of these works suffer the variability derived from enrolling patients in different stages of the disease and from the unknown effects of pharmacological treatments.

Here we present the largest study to date of sporadic PD patients at the early stage of the disease (de novo) and before any specific pharmacological treatment (drug-naïve) to perform gene expression profiling using Affymetrix microarrays on peripheral blood samples.

We show early changes in genes and pathways that provide new candidates on the quest for peripheral biomarkers of PD for diagnosis and patients' classification. Furthermore, we confirm differences in expression for biological pathways and selected genes previously identified in both PD blood and post-mortem brains increasing their significance as peripheral biomarkers. Together with the expected alteration in biological terms comprising neuronal apoptosis, mitochondrial dysfunction and inflammation, we have also found enrichment in genes involved in chromatin remodeling suggesting new strategies for pharmacological intervention.

\section{Methods}

Subjects

The study was approved by the Ethical Committee at the Movement Disorders Center of the Neurology Clinic, Azienda Ospedaliero-Universitaria Ospedali Riuniti, Trieste, Italy. Study participants gave written informed consent. During a two-year period we enrolled 52 patients with a first clinical diagnosis of PD, according to the UK Parkinson's Disease Society Brain Bank criteria. Thirty-two healthy age- and ethnicity-matched control subjects $(\mathrm{HC})$ were also included in the study. The "Discovery set" of the experiment included $40 \mathrm{PD}$ patients (68.8 years $\pm 6.9 \mathrm{SD})$ and $20 \mathrm{HC}$ (60.3 years \pm 5.7 SD). Subjects of the "Validation set" included $12 \mathrm{PD}$ (68.8 years $\pm 5.2 \mathrm{SD})$ and $12 \mathrm{HC}(68.0$ years $\pm 1.5 \mathrm{SD})$ volunteers. The demographic, clinical and haematological characteristics of the two study groups are shown in Table 1. De novo and drug-naïve PD patients were the selected cohort of this study. While both sustained denervation at the nigrostriatal dopaminergic axis, treatment with levodopa or dopamine agonist might interfere with different central neurotransmitter pathways influencing gene expression profiles. Therefore enrolled subjects did not take any centrally acting drugs in the previous 6 months. Although no genetic testing was performed, any family history of PD was determined by self-report and review of medical records if available was used as an exclusion criterion in order to minimize the inclusion of genetic forms of the disease. Inclusion criteria for $\mathrm{HCs}$ were no personal or familiar history of any neurological and psychiatric disorder.

\section{Clinical assessment}

Data referring to detailed history of the disease symptoms, co-morbid conditions, previous drug intake, and any evidence of family history of neurological diseases were collected. After a standardized neurological examination, Parkinsonian symptomatology was assessed by the motor subsection of the Unified Parkinson's Disease Rating Scale, UPDRS-motor part [12], and the Hoehn and Yahr staging scale [13]. Patients' cognition was assessed by the Mini-Mental State Examination (MMSE) [14]. Patients also underwent brain Computed Tomography and Magnetic Resonance Image scanning and Single Photon Emission Computed Tomography (SPECT) imaging with the pre-synaptic DA ligand ${ }^{123} \mathrm{I}-2 \beta$-carbometoxy-3 $\beta$-(4-iodophenyl)-N-(3-fluoropropyl) nortropane $\left({ }^{123}\right.$ I-FP-CIT) to assess the loss of nigrostriatal terminals and to gain a functional picture of the progression of the degenerative process within nigral DA neurons. SPECT images were classified as normal (symmetric bilateral uptake of the basal ganglia regions) or abnormal by visual inspection of an experienced Nuclear Medicine Specialist. Abnormal scans were graded as follow: asymmetric uptake with normal or almost normal putamen activity in one hemisphere and more marked changes on the other side (type 1); greatly reduced uptake in the putamen on both the right and left sides (type 2); very low uptake in the basal ganglia regions with increased specific background signal (type 3) [15].

\section{Blood collection, RNA purification and microarray processing} Blood samples were harvested directly and sequentially into 8 PAXgene Blood RNA tubes (PreAnalytiX, Hombrechtikon, $\mathrm{CH}$ ) via a 21 -gauge butterfly needle and then frozen and kept at $-80{ }^{\circ} \mathrm{C}$. Total RNA was purified using PAXgene ${ }^{\mathrm{rm}}$ Blood RNA kit (PreAnalytiX GmbH, Qiagen, Hilden, Germany) and DNaseI treatment was performed by 'on-column' treatment as recommended by manufacturer's instructions plus a second treatment subsequent to elution. RNA was then purified using 
RNeasy (Qiagen, Hilden, Germany) and quantified by NanoDrop ND-100 Spectrophotometer (NanoDropTechnologies; Wilmington, DE). RNA integrity was determined with 2100 Bioanalyzer (Agilent Technologies, Palo Alto, CA) and exclusively samples with $\mathrm{RIN} \geq 8$ were included in the subsequent investigations. Hybridization targets were synthesized with Ovation ${ }^{\text {Tw }}$ Whole Blood Solution (NuGEN) after comparison with other 2 methods (Additional file 1) and hybridized to HG-U133A 2.0 arrays (Affymetrix, Santa Clara, CA), investigating the expression of 18400 transcripts.

\section{Data analysis}

Principal Component Analysis (PCA) [16] was employed to reduce the dimensionality of the dataset before the application of classification methods. Partial Least Square Discriminant Analysis (PLS-DA) [17] was applied for classification purposes to obtain a first selection of the discriminating variables by using a binary coded $Y$ variable $(-1$ for control samples and +1 for pathological samples). A preliminary application of PLS-DA reduced the number of relevant variables to 1612 . Only classification models with a maximum of 6 Principal Component (PCs) were

Table 1 Demographics, clinical phenotypes and haematological values for de novo and drug-naïve PD patients and healthy controls

\begin{tabular}{|c|c|c|c|c|}
\hline \multirow[t]{2}{*}{ Diagnostic group } & \multicolumn{2}{|l|}{ Parkinson's disease } & \multicolumn{2}{|l|}{ Controls } \\
\hline & Discovery set & Validation set & Discovery set & Validation set \\
\hline Number & 40 & 12 & 20 & 12 \\
\hline Gender (Male/Female), number & 22/18 & $6 / 6$ & $10 / 10$ & $5 / 7$ \\
\hline Age, yr, mean (SD; range) & $68.83(6.94 ; 51-78)$ & $68.82(5.22 ; 59-76)$ & $60.30(5.69 ; 53-69)$ & $68.00(1.5 ; 65-71)$ \\
\hline Age at symptoms onset, $\mathrm{yr}$, mean (SD; range) & $67.65(6.80 ; 50-76)$ & $67.63(5.05 ; 58-74)$ & NA & NA \\
\hline Symptom duration, yr, mean (SD; range) & $1.14(0.66 ; 0.5-3)$ & $1.49(0.85 ; 0.5-3)$ & NA & NA \\
\hline \multicolumn{5}{|l|}{ Clinical phenotype at enrollment: } \\
\hline Unilateral symptoms, number (\%) & $24(60)$ & $10(83.3)$ & NA & NA \\
\hline Bilateral symptoms, number (\%) & $16(40)$ & $2(16.7)$ & NA & NA \\
\hline Tremor, number (\%) & $7(17.5)$ & $3(25.0)$ & NA & NA \\
\hline Bradykinesia/rigidity, number (\%) & $10(25.0)$ & $3(25.0)$ & NA & NA \\
\hline Mixed, number (\%) & $23(57.5)$ & $6(50.0)$ & NA & NA \\
\hline Hoehn and Yahr stage, mean (SD; range) & $1.45(0.55 ; 1-2)$ & $1.50(0.71 ; 1-3)$ & NA & NA \\
\hline UPDRS motor score, mean (SD; range) & $14.72(8.15 ; 3-29)$ & $13.82(7.22 ; 1-24)$ & NA & NA \\
\hline MMSE score, mean (SD; range) & $29.77(0.48 ; 29-30)$ & $29.73(0.90 ; 27-30)$ & NA & NA \\
\hline \multicolumn{5}{|l|}{${ }^{123}$ I-FP-CIT SPECT abnormality: } \\
\hline Type 1, number (\%) & $21(55.3)$ & 6 & NA & NA \\
\hline Type 2, number (\%) & $17(44.7)$ & 5 & NA & NA \\
\hline Not executed, number & 2 & 1 & NA & NA \\
\hline \multicolumn{5}{|l|}{ Haematological values (mean, SD): } \\
\hline White blood cells $\left[10^{A} 3 / \wedge \mathrm{L}\right]$ & $6.19(1.58)$ & $4.97(1.47)$ & $5.76(1.24)$ & $5.78(1.09)$ \\
\hline Neutrophils (\%) & $61.67(8.18)$ & $59.35(14.64)$ & $57.52(6.18)$ & $57.74(5.85)$ \\
\hline Lymphocytes (\%) & $27.85(7.49)$ & $28.92(2.72)$ & $31.31(6.52)$ & $30.63(6.21)$ \\
\hline Monocytes (\%) & $7.40(1.73)$ & $8.50(2.72)$ & $7.38(1.74)$ & $8.73(2.42)$ \\
\hline Eosinophils (\%) & $2.62(1.52)$ & $2.60(1.89)$ & $3.17(1.81)$ & $2.42(1.24)$ \\
\hline Basophils (\%) & $0.49(0.16)$ & $0.67(0.36)$ & $0.63(0.26)$ & $0.63(0.20)$ \\
\hline Red blood cells $[10 \mathrm{~A} 6 / \wedge \mathrm{L}]$ & $4.59(0.49)$ & $4.44(0.55)$ & $4.79(0.44)$ & $4.74(0.44)$ \\
\hline Hemoglobin [g/dL] & $14.40(1.23)$ & $14.28(1.49))$ & $14.14(0.88)$ & $14.85(0.52)$ \\
\hline $\mathrm{MCV} f \mathrm{fl}$ & $91.44(4.61)$ & $94.05(3.03)$ & $88.56(4.54)$ & $94.13(5.37)$ \\
\hline $\mathrm{MCH}[\mathrm{pg}]$ & $31.53(2.42)$ & $32.35(1.47)$ & $30.16(1.77)$ & $32.08(2.10)$ \\
\hline $\mathrm{MCHC}[\mathrm{g} / \mathrm{dl}]$ & $34.46(1.22)$ & $34.37(0.59)$ & $34.05(0.76)$ & $34.05(0.51)$ \\
\hline Platelet count $[10 \mathrm{~A} 3 / \wedge \mathrm{L}]$ & $226.71(66.72)$ & $176.33(14.87)$ & $221.60(43.62)$ & $226.92(78.05)$ \\
\hline
\end{tabular}


considered (Additional file 2). Ranking-Principal Component Analysis (R-PCA) [18] ranked variables according to their decreasing discriminant ability. Linear Discriminant Analysis (LDA) [19], a Bayesian classification method, provided the classification of the samples considering the multivariate structure of the data. Here, a Forward Selection procedure [19] was applied to the principal components. The classification performance of the models was evaluated by the non-error rate (NER\%), namely the percentage of overall correct assignments. Further data processing was performed in the $\mathrm{R}$ computing environment (http://www.r-project.org/) version 2.8.0 with BioConductor packages (http://www.bioconductor.org/). Data were first filtered by eliminating probes with detection call of poor quality as well as those with intensity value lower than $\log _{2} 100$ for all the samples. Of the original 60 samples, one (a control samples) did not pass the microarray hybridization quality controls and was excluded from further analyses. Therefore, the final dataset consisted of 59 samples described by 15137 probes.

Robust Multi-Array Average (RMA) normalization was applied to microarray data and these were imported in the Multiexperiment Viewer $(\mathrm{MeV})$ software version 4.5.1 for Windows XP (http://www.tm4.org/mev.html). Statistical analysis was performed with PUMA [20], SAM (Significance Analysis of Microarrays) [21] and Rank Product (RP) modules [22] to detect significantly differentially expressed genes.

PUMA is a Bayesian method (available in R BioConductor) that includes probe-level measurement error into the estimates of expression profile [20]. These were normalized through a median global array scaling, and a single expression value for each condition was combined from the replicates and associated to a probability of positive log ratio (PPLR) between conditions. In order to facilitate the interpretation of results, PPLR was converted in a p-value-like form: 1-PPLR was used for upregulated genes while PPLR for down-regulated ones. SAM was chosen for its power to allow the control of false positive results (False Discovery Rate or FDR). This is particularly relevant when looking at human samples because of the inherent rate of genetic variation among individuals. Data were filtered so that only probe sets that had a Present call and intensity value of $>100$ in at least half the arrays of the smaller group were retained.

Functional analyses were performed using Gene Ontology (GO) annotations [23], DAVID Bioinformatics Resources [24] and Gene Set Enrichment Analysis (GSEA) [25] as implemented at http://www.broadinstitute.org/ gsea/, version 2.06 .

\section{Quantitative real-time PCR (RT-qPCR)}

Total RNA was reverse-transcribed using Superscript III Reverse Transcriptase (Invitrogen, Carlsbad, CA, USA),
$25 \mathrm{ng}$ random hexamers and $2.5 \mu \mathrm{M}$ oligo (dT) 20 primers according to the manufacturer's recommendations. Real-time PCR was performed in the presence of $1.25 \mu \mathrm{L}$ of cDNA template, TaqMan ${ }^{\circ}$ gene expression master mix and commercially available $\mathrm{TaqMan}^{\circ}$ gene expression assays. It was run on ABI Prism ${ }^{\circ} 7900 \mathrm{HT}$ Sequence Detection System (Applied Biosystems, Foster City, CA, USA). TaqMan assays were: CBX5 (Hs0112 7577_m1), TCF3 (Hs00413032_m1), DOCK10 (Hs0039 1515_m1), MAN1C1 (Hs00220595_m1), ALDH1A1 (Hs 00946916_m1), HSPA8 (Hs03044880_gH), PSMC4 (Hs0 0197826_m1), HIP2 (Hs00193507_m1), SKP1 (Hs00 749532_s1), PGK1 (Hs99999906_m1) and UBC (Hs00 824723_m1). Thermal cycler conditions were as follows: $50{ }^{\circ} \mathrm{C}$ for $2 \mathrm{~min}$ and $95{ }^{\circ} \mathrm{C}$ for $10 \mathrm{~min}$, followed by 40 cycles of amplification at $95{ }^{\circ} \mathrm{C}$ for $15 \mathrm{~s}$ and $60{ }^{\circ} \mathrm{C}$ for $1 \mathrm{~min}$. Amplification efficiencies were higher than $90 \%$ for each primer set. Reactions were run in duplicate and a replica was performed. Negative controls, such as nontemplates wells as negative reverse transcriptase controls, were assembled to rule out respectively DNA cross contamination of the reagents and genomic DNA in the samples. The amplified products were separated on a $2 \%$ agarose gel and visualized with ethidium bromide staining. To identify the best candidate genes as endogenous controls for normalization, $8 \mathrm{PD}$ and $8 \mathrm{HC}$ age-matched peripheral blood samples were analyzed using the TaqMan ${ }^{\circ}$ array human endogenous control Cards (Applied Biosystems, Foster City, CA, USA). As the best reference genes, PGK1 and UBC were similarly selected by geNorm and Normfinder applications. The relative gene expression was evaluated by normalization to the geometric mean of the 2 selected endogenous controls and a pool of HC samples was used as calibrator. Statistical analysis relied on Qbase plus software (SPSS Ltd., UK) and graphs were generated with Graphpad Prism 6.0 (Graphpad Software Inc, USA).

\section{Availability of supporting data}

Microarray data sets are available in the Gene Expression Omnibus (GEO; http://www.ncbi.nlm.nih.gov/projects/geo/) with Accession Number GSE72267. Suppor ting data (Additional files 1, 2, 3, 4, 5, 6, 7, 8, 9, 10, 11, 12,13 and 14) are included in the article.

\section{Results}

\section{Study design}

Here we aim to identify gene expression patterns in peripheral blood of de novo and drug-naïve PD patients by comparing 40 sporadic PD versus $20 \mathrm{HCs}$ ("Discovery set"). To this purpose, patients were enrolled at the early clinical stage of the disease as evaluated by a neurologist. Subjects did not take any centrally acting drugs in the previous 6 months. Table 1 shows the demographic and clinical characteristics of all enrolled subjects. As expected, 
most patients showed a prevalent asymmetric parkinsonian symptomatology that, in the majority of cases, comprised the classical triad of tremor, bradykinesia, and rigidity. Accordingly, an asymmetric reduction of striatal activity on the ${ }^{123}$ I-FP-CIT SPECT images was observed. The prevalent case consisted of a putaminal alteration contralateral to the clinical most affected side.

Experiments were carried out aiming to optimize the protocol for blood transcriptomics to satisfy the required criteria for biomarker discovery [4]. Special attention was devoted to assess the effects on gene expression profiles of patient's physiological status at the time of collection and of storage conditions of biological samples (data not shown). After comparing three methods for the synthesis of microarray hybridization targets, the Ovation ${ }^{\mathrm{TM}}$ Whole Blood Solution (NuGEN) was chosen for its high sensitivity (three times increase in Present call) and robust reproducibility (Additional file 1). Blood was collected from study subjects into PAXgene Blood RNA tubes (PreAnalytiX, Hombrechtikon, $\mathrm{CH}$ ) after a fasting period and at the same time of the day to limit circadian-dependent variability. Furthermore, hematological values were determinated to exclude any significant difference among blood cell types that could affect RNA composition. Total RNA was purified using PAXgene ${ }^{\mathrm{TM}}$ Blood RNA kit (PreAnalytiX GmbH, Qiagen, Hilden, Germany) and underwent two DNaseI digestion steps. Only high quality RNAs (RNA Integrity Number or RIN $\geq 8$ ) were included in the study as determined with 2100 Bioanalyzer (Agilent Technologies, Palo Alto, CA). Hybridization targets were synthesized with Ovation ${ }^{\text {Tw }}$ Whole Blood Solution (NuGEN) and hybridized to HG-U133A 2.0 arrays (Affymetrix, Santa Clara, CA), investigating the expression of 18400 transcripts. For details, see Material and methods.

\section{A gene panel discriminates PD patients from HCs}

A statistical analysis was carried out to identify a gene panel able to discriminate between patients and controls. To this purpose we took advantage of R-PCA [18] to select the most discriminant variables followed by LDA to obtain the best classification model with a maximum of 6 PCs (Additional file 2). Thus 395 variables were selected (Additional file 3) and a model including five highly significant $\mathrm{PCs}(p$-levels $<0.01) \quad\left(\mathrm{PC}_{2}, \mathrm{PC}_{6}, \mathrm{PC}_{3}\right.$, $\mathrm{PC}_{5}, \mathrm{PC}_{1}$ ) correctly classified all the samples (Fig. 1a). The squared Mahalanobis distance (Additional file 4) of each sample from the centroid of its own and of the other class showed that the classification performance was very robust, the only exception being sample P041BB01 characterized by similar distances from the two classes.

We then analyzed the set of 395 variables, identified by 466 probes, for biological processes significantly enriched in the panel. As shown in Fig. 1b and in Additional file 5, processes such as "regulation of apoptosis", "lymphocyte activation", "leukocyte activation" and "lipid biosynthetic process" were found. These expected findings were already previously associated to PD in blood and post-mortem brains confirming the experimental and bioinformatic pipeline. Moreover, we originally identified several GO terms associated to epigenetic remodeling including "chromatin assembly or disassembly" (GO:0006333).

\section{Differential gene expression analyses of blood samples of drug-naïve sporadic PD patients}

To identify differentially expressed genes between sporadic PD patients and controls, analysis of gene expression profiles was performed with different bioinformatics algorithms. First, we applied a recently introduced probabilistic model (PUMA) [20] to estimate fold changes and their significance for each probe on the array. No filters were applied to the dataset. By selecting an arbitrary threshold of $1 \%$ probability of including false positives, 306 differentially expressed probes between the two groups were identified corresponding to 282 unique genes (Additional file 6). We also carried out analysis with the parametric test SAM [21] (Additional file 7) and with the non-parametric RP [22] (Additional file 8). These analyses showed respectively 107 and 280 genes common with the ones obtained with PUMA.

Importantly, 54 genes were identified with R-PCA, PUMA and SAM representing a list of candidates for biomarker discovery (Additional file 9).

\section{Tissue expression and functional analysis of differentially expressed genes}

A tissue enrichment analysis was then carried out on the list of differentially expressed genes obtained with PUMA. Surprisingly, $50 \%$ of them were enriched in brain [24] (Table 2a). Furthermore, we compared them to those genes expressed in the DA neurons of the $\mathrm{SN}$, the key site of degeneration in PD. To this purpose, we took advantage of the gene expression profile of mouse DA neurons in the SN that we have recently obtained by coupling transgenic labelling, Laser Capture Microdissection and Affymetrix expression analysis [26-28]. After converting the mouse gene annotation to human annotation, 135 genes were common to the 282 genes identified with the co-expression analysis (Additional file 10). These findings were confirmed for the list of genes obtained with SAM (data not shown) and prove an extensive overlap between genes expressed in mouse DA neurons and those differentially expressed in the blood of PD patients.

We then took advantage of Gene Ontology and GSEA to identify enriched biological pathways (Additional 11). As expected from previous studies both in PD blood and post-mortem brains, the most significant terms include "lymphocyte activation", "lipid homeostasis", "midbrain 
development" and "leukocyte activation". Importantly, we also identified for the first time a strong enrichment in epigenetic-related Gene Ontology terms such "regulation of gene expression, epigenetic" and "chromatin assembly or disassembly" (Table $2 \mathrm{~b}$ ). These processes were also enriched in the list of differentially expressed genes obtained with SAM (Additional file 7). To validate the involvement of transcripts associated to chromatin remodeling and methylation-dependent processes, blood samples from the "Discovery set" were tested with RTqPCR assays for the differentially regulated CBX5, HELLS and MECP2 mRNAs as well as for ASFA1,

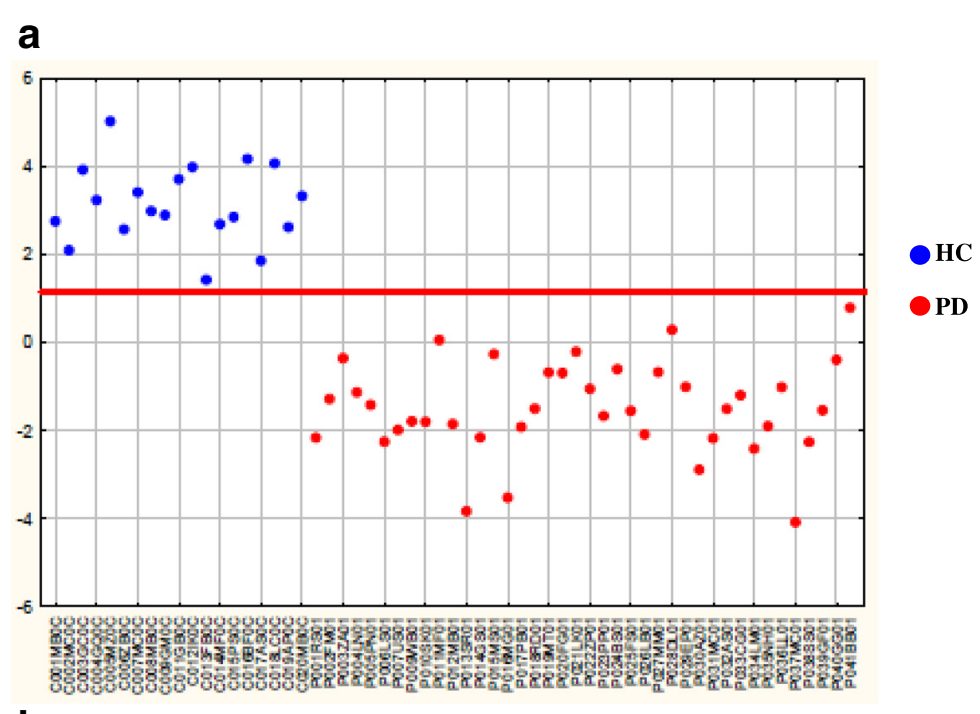

b

\begin{tabular}{|l|l|}
\hline Term & P-Value \\
\hline GO:0006333 chromatin assembly or disassembly & $3.23 \mathrm{E}-04$ \\
\hline GO:0042981 regulation of apoptosis & $1.42 \mathrm{E}-03$ \\
\hline GO:0043067 regulation of programmed cell death & $1.65 \mathrm{E}-03$ \\
\hline GO:0043065 positive regulation of apoptosis & $1.72 \mathrm{E}-03$ \\
\hline GO:0010941 regulation of cell death & $1.74 \mathrm{E}-03$ \\
\hline GO:0044092 negative regulation of molecular function & $1.75 \mathrm{E}-03$ \\
\hline GO:0043068 positive regulation of programmed cell death & $1.86 \mathrm{E}-03$ \\
\hline GO:0010942 positive regulation of cell death & $1.96 \mathrm{E}-03$ \\
\hline GO:0001775 cell activation & $7.29 \mathrm{E}-03$ \\
\hline GO:0043549 regulation of kinase activity & $8.00 \mathrm{E}-03$ \\
\hline GO:0046649 lymphocyte activation & $8.96 \mathrm{E}-03$ \\
\hline GO:0042325 regulation of phosphorylation & $8.99 \mathrm{E}-03$ \\
\hline GO:0031328 positive regulation of cellular biosynthetic process & $9.04 \mathrm{E}-03$ \\
\hline GO:0009891 positive regulation of biosynthetic process & $1.07 \mathrm{E}-02$ \\
\hline GO:0051338 regulation of transferase activity & $1.15 \mathrm{E}-02$ \\
\hline GO:0010604 positive regulation of macromolecule metabolic process & $1.22 \mathrm{E}-02$ \\
\hline GO:0045321 leukocyte activation & $1.28 \mathrm{E}-02$ \\
\hline GO:0051174 regulation of phosphorus metabolic process & $1.31 \mathrm{E}-02$ \\
\hline GO:0019220 regulation of phosphate metabolic process & $1.31 \mathrm{E}-02$ \\
\hline GO:0043086 negative regulation of catalytic activity & $1.35 \mathrm{E}-02$ \\
\hline GO:0010648 negative regulation of cell communication & $1.51 \mathrm{E}-02$ \\
\hline GO:0008219 cell death & $1.55 \mathrm{E}-02$ \\
\hline GO:0016265 death & $1.64 \mathrm{E}-02$ \\
\hline GO:0006917 induction of apoptosis & $1.68 \mathrm{E}-02$ \\
\hline GO:0012501 programmed cell death & $1.71 \mathrm{E}-02$ \\
\hline GO:0012502 induction of programmed cell death & $1.73 \mathrm{E}-02$ \\
\hline GO:0009968 negative regulation of signal transduction & $1.76 \mathrm{E}-02$ \\
\hline GO:0008610 lipid biosynthetic process & $1.81 \mathrm{E}-02$ \\
\hline GO:0010557 positive regulation of macromolecule biosynthetic process & $1.83 \mathrm{E}-02$ \\
\hline
\end{tabular}

Fig. 1 Ranking-PCA applied to the 395 selected variables to compare PD patients and HCs. a. Representation of the samples along the first canonical root. The first canonical root (y-axis) is reported for each sample (x-axis). Blue circles correspond to control samples while red circles to pathological samples. The solid line represents the separation between the two classes. The variables are reported in Additional file 3 in the order in which they are included in the model. b. Functional annotation analysis of the 395 variables. Over-represented GO annotations with at least 10 genes and $P<0.02$ (Fisher exact probability) are presented. The complete data of enriched GO annotations are available in Additional file 5 
Table 2 Identification of tissue-enrichment terms and biological processes associated with selected genes dysregulated in blood of PD patients versus controls

\begin{tabular}{|c|c|c|c|c|c|}
\hline Category & Term & Count & Percent & $P$-Value & Benjamini \\
\hline \multicolumn{6}{|l|}{$a$} \\
\hline UP TISSUE & Brain & 141 & 50.00 & $7.4 \mathrm{E}-3$ & 7.1E-1 \\
\hline UP TISSUE & Testis & 70 & 24.08 & $1.9 \mathrm{E}-2$ & $8.0 \mathrm{E}-1$ \\
\hline UP TISSUE & Epithelium & 59 & 20.09 & $3.2 \mathrm{E}-2$ & 8.4E-1 \\
\hline UP TISSUE & Lymphoma & 4 & 1.04 & $6.1 \mathrm{E}-2$ & 9.3E-1 \\
\hline UP TISSUE & Testicle & 3 & 1.01 & $7.4 \mathrm{E}-2$ & $9.2 \mathrm{E}-1$ \\
\hline \multicolumn{6}{|l|}{ b } \\
\hline GOTERM_BP_FAT & ncRNA processing & 10 & 3.05 & $4.2 \mathrm{E}-3$ & 1.0EO \\
\hline GOTERM_BP_FAT & regulation of gene expression, epigenetic & 6 & 2.01 & $1.1 \mathrm{E}-2$ & 1.0EO \\
\hline GOTERM_BP_FAT & lymphocyte activation & 10 & 3.05 & $1.3 \mathrm{E}-2$ & 1.0EO \\
\hline GOTERM_BP_FAT & lipid homeostasis & 5 & 1.08 & $1.5 \mathrm{E}-2$ & 1.0EO \\
\hline GOTERM_BP_FAT & chromatin assembly or disassembly & 7 & 2.05 & 1.7E-2 & $9.9 \mathrm{E}-1$ \\
\hline GOTERM_BP_FAT & midbrain development & 3 & 1.01 & $2.0 \mathrm{E}-2$ & $9.9 \mathrm{E}-1$ \\
\hline GOTERM_BP_FAT & chemical homeostasis & 17 & 6.00 & $2.9 \mathrm{E}-2$ & 1.0EO \\
\hline GOTERM_BP_FAT & negative regulation of protein kinase cascade & 4 & 1.04 & $3.0 \mathrm{E}-2$ & 1.0EO \\
\hline GOTERM_BP_FAT & ncRNA metabolic process & 9 & 3.02 & $3.5 \mathrm{E}-2$ & $1.0 \mathrm{EO}$ \\
\hline GOTERM_BP_FAT & regulation of action potential & 5 & 1.08 & $3.8 \mathrm{E}-2$ & 1.0EO \\
\hline GOTERM_BP_FAT & leukocyte activation & 10 & 3.05 & $3.9 \mathrm{E}-2$ & 1.0EO \\
\hline GOTERM_BP_FAT & cell activation & 11 & 3.09 & $4.2 \mathrm{E}-2$ & 1.0EO \\
\hline
\end{tabular}

a)Tissue enrichment analysis. Count: number of genes involved in the term; \%: percentage of involved genes/total genes; $P$-Value: modified fisher exact $P$-value, EASE Score; Benjamini: adjusted P-value using Benjamini-Hochberg procedure. b)Biological processes identified by GO annotations (DAVID)

DNMT3A and PRMT1 as part of the very same GO gene list (Additional file 11). As shown in Additional file 12, their differential expression was confirmed.

\section{Validation of selected differentially expressed genes}

We carried out RT-qPCR on additional un-profiled samples to independently assess expression changes for a selected group of genes obtained from the array data. Blood samples were collected from 24 sex-, age- and ethnicity-matched subjects: 12 sporadic PD (68.8 years \pm $5.2 \mathrm{SD})$ and $12 \mathrm{HC}$ (68.0 years $\pm 1.5 \mathrm{SD}$ ) ("Validation set"). Enrolment inclusion criteria and procedures were as those defined for the "Discovery set". Clinical and demographic characteristics of subjects are reported in Table 1.

Genes for validation were selected from the candidates list of biomarkers commonly obtained with R-PCA, PUMA and SAM analysis (Additional file 9). Four transcripts were chosen among the ones with the most significant $P$-values $(p<0.0003)$ and tested with RT-qPCR. Data were normalized to the geometric mean of PGK and $\mathrm{UBC}$, the most reliable reference genes. These were identified using $\operatorname{TaqMan}^{\circ}$ array human endogenous control cards (Applied Biosystems, Foster City, CA, USA) as reported in Materials and methods section and in Additional file 13. As shown in Fig. 2a, statistically significant results from RT-qPCR analysis were achieved for Chromobox homolog 5 (CBX5) $(P=2.88 \mathrm{E}-02)$, Transcription factor 3 (TCF3) $(P=5.12 \mathrm{E}-04)$, Dedicator of cytokinesis 10 (DOCK10) $(P=1.52 \mathrm{E}-02)$ and Mannosidase, alpha class $1 \mathrm{C}$ (MAN1C1) $(P=1.15 \mathrm{E}-03)$. Overall, these RT-qPCR data validate expression changes identified through array analysis with a different technology and on an independent set of samples.

To correlate these findings with genes previously identified as differentially expressed in PD post-mortem brains and blood [29, 30], we have found a significant difference of expression in the very same "Validation set" for Aldehyde Dehydrogenase 1 Family, member A (ALDH1A1) $(P=1.51 \mathrm{E}-02)$, Proteasome (prosome, macropain) $26 \mathrm{~S}$ subunit PSMC4 $(P=1.80 \mathrm{E}-03)$ and Heat shock $70 \mathrm{kDa}$ protein 8 (HSPA8) $(P=8.20 \mathrm{E}-03)$ but not for Huntingtin interacting protein 2 /ubiquitin-conjugating enzyme E2K (HIP2/UBE2K) $(P=3.00 \mathrm{E}-04)$ (Fig. 2b).

\section{Drug network analysis of PD-associated genes}

In the search for potential new PD treatments we investigated whether FDA-approved drugs could elicit a transcriptional profile similar or opposite to the one observed in peripheral blood of PD patients. This approach is based on the observation that a large portion of differentially expressed genes in PD blood is expressed in mesencephalic DA neurons and that altered GO biological terms are 

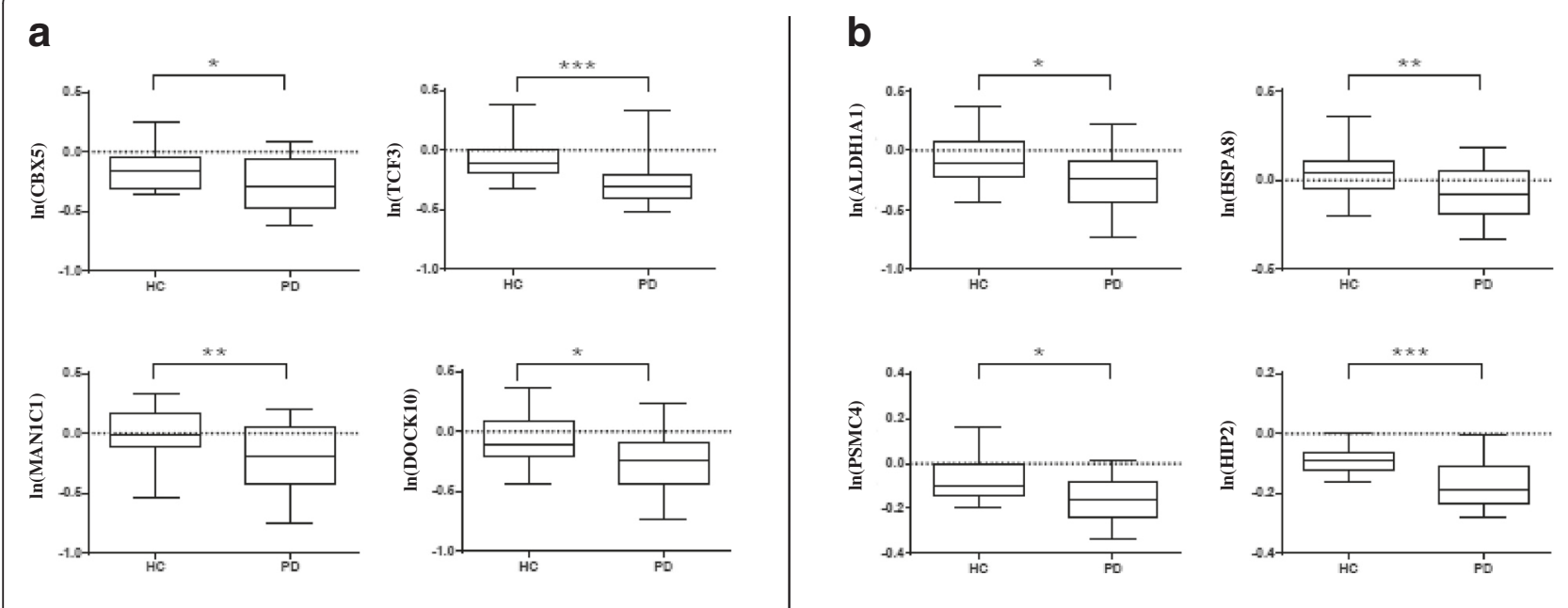

Fig. 2 RT-qPCR validation experiments of selected transcripts. The box plots show the natural logarithms of the relative gene expression levels (calculated by dividing the RT-qPCR values by the geometric mean of the HKs PGK1 and UBC raw quantities) for the individual genes (a and $\mathbf{b})$ in blood samples of 12 de novo PD patients and 12 age- and sex-matched HCs. The statistical significance was calculated by t-test $\left(^{*} p<0.05\right.$,

$\left.{ }^{* *} p<0.01,{ }^{* * *} p<0.001\right)$

common in PD blood and post-mortem brains. To this purpose we took advantage of a new approach to identify drug mode of action from gene expression profiles [31]. Specifically, drugs are connected in a network if they elicit a similar transcriptional response according to a new similarity measure based on a modification of GSEA [25]. The drug network consists of 1309 compounds that can be subdivided in 106 communities of drugs, i.e. groups of drugs very similar to each other with a similar mode of action but very different from other drugs in the network. To investigate which drugs trigger the most similar response to the changes found in PD patients, genes were ranked according to their differential expression in PD versus control and the network was queried. The drugs sorted according to their similarity to PD are shown in the Additional file 14. Interestingly, as reported in Fig. 3a, several antipsychotic drugs elicited a transcriptional profile similar to PD (Community 100, $P=3.83 \times 10-6$ considering the top-ranked 35 similar to PD drugs and $P=6.76 \times 10-9$ considering the top-ranked 100 ones). On the other hand, examining the drugs which elicit an "anti-similar" transcriptional response (i.e. which up-regulate genes found

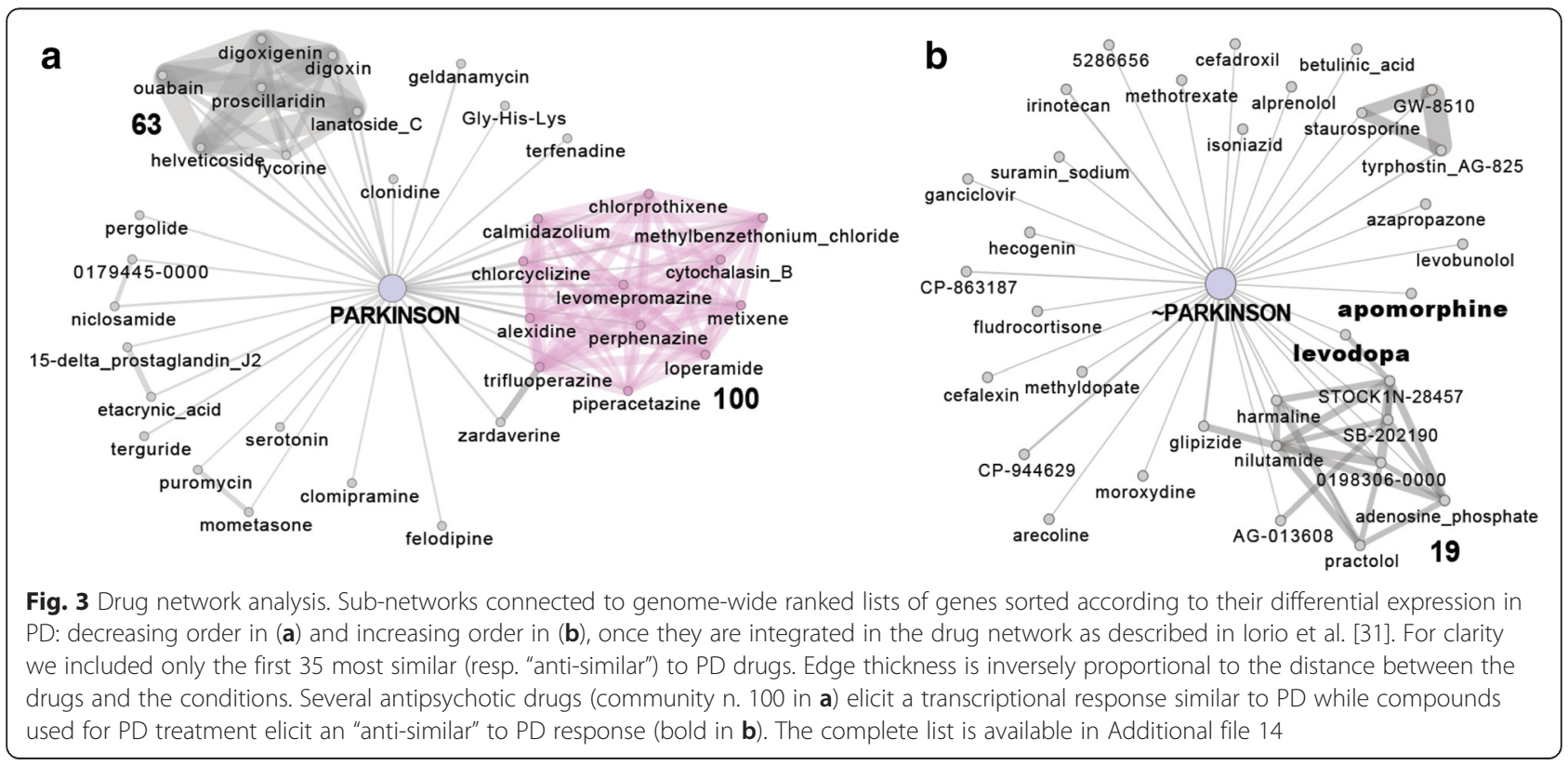


down-regulated in PD and vice-versa), it is noteworthy that apomorphine and levodopa occupy top-ranked positions and are currently used for treating PD (Fig. 3b and Additional file 14).

\section{Discussion}

This work is the largest study to date of the peripheral whole blood transcriptome of drug-naïve sporadic PD patients at the time of their first diagnosis. A gene panel discriminates PD patients from healthy controls. While we confirmed alterations previously found in PD blood and post-mortem brains such as neuronal apoptosis, lymphocyte activation and mitochondrial dysfunction, we have also unveiled changes enriched in biological terms related to epigenetic modifications including chromatin remodeling and methylation.

A correct diagnosis at the early stages of the disease remains an unresolved and crucial issue in PD. This is important to choose the best medical treatment from disease onset and to apply the best criteria for patient selection and enrollment in clinical trials. The potential of blood as a surrogate tissue in PD is under intense scrutiny and blood transcriptomics is expected to facilitate the identification of biomarkers for early diagnosis and drug discovery [32].

A short but significant list of recent microarraybased studies has used human blood as RNA source to look for differentially expressed genes in sporadic and genetic PD patients [5-11]. In the pioneering work by Scherzer et al. [5], 22 genes were identified with microarray profiling of whole blood of 50 PD patients, among which 9 de novo subjects. A risk marker given by 8 genes (VDR, HIP2, CLTB, FPRL2, CA12, CEACAM4, ACRV1, and UTX) predicted PD and was not biased by dopamine replacement therapy. The analysis of a genetically homogenous population of 88 Ashkenazi patients [6], including 20 de novo, evidenced for the first time the decreased expression of $\mathrm{B}$ cells-related genes in PD. Karlssonn et al. [8] analyzed samples from 79 PD subjects at different stages of the disease, including 23 de novo patients and relative controls, proposing a classifier predicting sporadic and de novo PD. LRPPRC, BCL2 and SRSF8 were shared with the 22 genes list as in Scherzer et al. [5]. Furthermore, it presented HSPA8 and UBE2K/HIP2 in common with Molochnikov et al. [30]. Potashkin et al. [7] took advantage of splice variant-specific microarrays to identify a biosignature composed of 13 mRNAs (c5orf4, wls, macf1, prg3, eftud2, pkm2, slc14a1-s, slc14a1-1, mpp1, copz1, znf160, map4k1 and znf134) whose expression is altered in peripheral blood of early-stage PD patients. Recently, they identified two novel longitudinally markers (HNF4 and PTB1) by means of network-based and transcriptomic meta-analyses [33]. Interestingly, gene expression analysis of peripheral blood mononuclear cells from 20 sporadic PD patients and 9 individuals, heterozygous for the LRRK2 G2019S mutation, showed deregulation of the immune system, endocytosis and eukaryotic initiation factor 2 signaling [11].

Although there are a number of promising gene signatures, blood transcriptomics have not yet delivered the expected results for biomarker discovery in PD. One of the major concerns is the scarce overlap among candidate genes lists of these studies. Variances in the procedures for collection, processing and analysis of samples may strongly limit the reproducibility of gene expression data. Importantly, these differences may also be explained in biological terms. First, genetic variations in human populations may lead to diversity in transcriptional changes in disease. Furthermore, the majority of these works analyzed peripheral blood samples from sporadic PD patients at different stages of the disease and under pharmacotherapy raising the questions of whether changes are related to the disease stage, therapy or both. Finally, it is now clear that PD is a systemic and a highly heterogeneous disease, as classified according to distinct clinical subtypes [34].

On the other hand, blood transcriptomics studies identify a common repertory of enriched GO biological terms as altered in PD. These include "neuronal apoptosis", "mitochondrial dysfunction", "leukocyte activation" and "deregulation of the immune system".

To overcome part of the limitations of previous analyses, our study design takes advantage of 1) a carefully selected drug-naïve and de novo ethnically-defined PD population size, 2) standardized, simple and well-defined technologies and methods whose protocols and procedures are generally recognized as robust and reproducible. Our purpose was to establish an optimized pipeline to collect biological samples for blood transcriptomics to minimize confounding signals such as artifacts of sample preparation and processing while maximizing reproducibility and sensitivity. Most importantly, the use of the NuGEN method for target preparation results in higher sensitivity (three times increase in Present calls) leading to a wider range of intensities and a smaller impact of technical variability.

By performing data analyses, we prove that gene expression profiling of peripheral blood discriminates patients from HCs. Furthermore, we identified a list of genes (Additional file 9) that were differentially expressed in PD patients at the onset of motor symptoms and before initiating any pharmacological treatment. Selected transcripts were validated in an independent cohort of patients and HCs ("Validation set") with a different technology (RT-qPCR) strengthening our findings.

A very limited overlap was found in the identities of single genes between our study and those previously 
published. This is not surprising and probably due to differences in the cohort of patients and in technical settings.

The level of expression changes between PD patients and $\mathrm{HC}$ was in the range of 20 to $50 \%$. While these values are similar to the ones identified in other blood transcriptomics studies, patients were at an early stage of the disease and differential expression may increase with disease progression. Furthermore, while differential expression may occur only in selected cells' populations, genes may be expressed in the majority of blood cell types decreasing fold changes measured in whole blood.

Our analyses confirmed the main biological GO terms found altered in previous blood transcriptomics of PD patients. Together with "neuronal apoptosis" and "mitochondrial dysfunction", differentially expressed pathways include "lymphocyte activation" and "leukocyte activation". Among the single validated transcripts, MAN1C1, mannosidase, alpha, class $1 \mathrm{C}$, member 1 is a protein partner of MAN1A2 previously identified by Scherzer et al. [5]. These two key $\alpha 1,2$-mannosidases catalyze the earliest steps of mannose removal required for the conversion of high mannose to hybrid and subsequently complex $\mathrm{N}$-glycans. Current hypothesis suggests a key role for core $\mathrm{N}$-glycan structures (i.e. mannose) in providing signals to the innate immunity system for recognizing cells during inflammation [35]. Transcription factor 3 (TCF3), also known as E2A, plays an important role in the development and differentiation of $\mathrm{B}$ and $\mathrm{T}$ lymphocytes [36]. It mainly functions as a transcriptional repressor [37] being counteracted at multiple levels by Wnt signaling [38]. This pathway is crucial in normal function and survival of midbrain DA neurons [39] and its alteration has been already reported in gene expression studies of peripheral blood of PD patients [7, 11]. DOCK10 (Dedicator of cytokinesis 10) is a gene that encodes a member of the zizimin subfamily belonging to the Dock protein family, comprising atypical Rho guanine nucleotide exchange factors for Rac and/or Cdc42 GTPases [40]. Dock10 may represent a point of convergence for IL-4 signaling and small Rho GTPase function in B cells [41] and this might be important for the wellknown dysregulation of IL-4 signaling in peripheral blood of sporadic PD [11].

Importantly, we make the original observation that crucial elements of chromatin remodeling and methyltransferase machineries are major targets of PD-associated molecular events in living patients suggesting a role of epigenetic regulation in neurodegeneration [42]. Recent data support epigenetic modulation in neurodegenerative diseases such as Alzheimer's, Huntington's and Amyotrophic Lateral Sclerosis. A direct relationship between epigenetics and PD has not been systematically assessed although sparse evidences are available. Dopamine depletion in PD is associated with a reduction in histone H3K4me3, whereas chronic levodopa therapy leads to deacetylation of histones H4K5, K8, K12, and K16. Treatment of animals with MPTP (1-methyl-4-phenyl-1,2,3,6- tetrahydropyridine), widely used as a PD model, induces $\mathrm{H} 3$ acetylation, which is reduced after treatment with levodopa [43]. Interestingly, methylation of SNCA intron 1 was found reduced in DNA from sporadic PD patients' $\mathrm{SN}$, putamen, and cortex, while its expression in a PD patient heterozygous for the A53T mutation was found to be monoallelic due to epigenetic silencing [44-46]. In fact, hypomethylation of SNCA and LRRK2 in leukocytes of peripheral blood has been suggested as a potential noninvasive biomarker for PD early diagnosis [47]. Most recently, $\alpha$-synuclein was found to trigger DNMT1 aberrant cytoplasmic localization in PD post-mortem brains and animal models leading to a global DNA hypomethylation. Furthermore, DNMT1 protein itself was reduced to $50 \%$ in post-mortem PD brains [48].

In this context an intriguing chain of events has been proposed linking mitochondrial dysfunction to epigenetic changes in PD [49-51]. The age-dependent downregulation of metallothioneins may render DA neurons susceptible to oxidative stress and Charnoly body formation, an early and universal mitochondrial biomarker of cell injury, apoptosis and progressive neurodegeneration. In turn, the formation of 8-hydroxy, 2-deoxyguanosine, a PD biomarker in urine, can affect the epigenetic status of nuclear DNA.

Currently, several epigenetic-based drugs are investigated as potential treatment strategies for PD, including HDAC and DNMT inhibitors [52-55]. Further work is needed in evaluating these promising therapeutics.

Chromobox homolog 5 (CBX5), also named HP1a, is the most differentially expressed gene in our analysis. It belongs to a class of multifunctional chromatin-associated adapter proteins present in constitutive heterochromatin. There it plays an essential role in establishing and maintaining heterochromatin-mediated gene silencing [56]. In addition to self-association, HP1-interacting partners include the DNA methyltransferase DNMT3A, which we have found decreased in PD patients. DNMT3A is required for de novo DNA methylation [57], and its activity has been related to several functions in the nervous system including neuronal differentiation, synaptic plasticity and memory formation. Microarray data also detect changes in the expression of other genes involved in chromatin remodeling and epigenetic regulation such as MECP2, an essential epigenetic regulator in human brain development that has been associated to activitydependent synaptic maturation [58], and ASF1A, a histone chaperone that participates in nucleosomes disassemblies and interacts with histone-acetylation-recognizing bromodomains [59]. Protein arginine methyltransferase (Prmt1), 
Thymocyte selection-associated high mobility group box (Tox), Enhancer of zeste homolog 1 (Ezh1), and Sin3Aassociated protein (SAP30) were also dysregulated.

Changes in peripheral blood seem to reflect molecular events in the brain. Selected genes and GO terms, identified as dysregulated in PD post-mortem brains, were previously confirmed as altered in the peripheral blood of living patients. In this context, Grunblatt et al. [29] carried out RT-qPCR in more than 100 medicated and 11 de novo PD patients to study 12 transcripts previously proved to be differentially expressed in PD post-mortem brains. Four of them were proposed as biomarkers for PD with a specificity of more than $80 \%$. Recently, Molochnikov et al. [30] presented a highly similar five-gene set (SKP1A, HIP2, ALDH1A1, PSMC4 and HSPA8) that differentiated early PD from HCs.

Here we show that expression of PD-associated genes in blood is enriched in brain tissue. Furthermore, about $50 \%$ of these genes are also expressed in DA neurons of the $\mathrm{SN}$, the key site of neurodegeneration in PD. As expected, the enriched GO terms of this gene expression study present a significant overlap with those unveiled in gene expression profiling of PD post-mortem brains. Consequently, we specifically investigated the expression of selected genes previously shown as differentially expressed in both PD post-mortem brains and blood [29, 30]. Considering that we could not observe gene expression changes for those genes since no Affymetrix ID probes were present on the chips used in this study, we took advantage of RT-qPCR assays to test their expression in the "Validation set" of samples. We thus confirmed a significant difference of expression for ALDH1A1, a detoxification enzyme that participates in the metabolism of catecholamines and plays a key role in the protection of the nigrostriatal DA neurons [60], for PSMC4, involved in the ubiquitin proteasome degradation pathway and for HSPA8 (Fig. 2b). Their differential expression further corroborates that events associated with degeneration in PD post-mortem brain can also be detected in peripheral blood of living patients.

These observations are substantially strengthened by our drug network analysis. Among the 106 communities that grouped 1309 compounds for their gene expression patterns, our study revealed that the "antipsychotic" drug community was significantly mimicking PD gene expression phenotypes. This community of drugs, acting at the level of DA neurotransmission, highlights that we are detecting changes in gene patterns relevant for CNS function in PD. In addition, drugs as apomorphine and levodopa, two molecules currently used in PD clinical treatment, are selected in the community of drugs that elicit an "antisimilar" transcriptional response.

\section{Conclusions}

To our knowledge, this is the largest study to date using whole peripheral blood to investigate early gene expression changes in drug-naïve and de novo sporadic PD patients. We are conscious that replication is needed in a larger well-characterized prospective study to confirm and define the clinical use of this set of transcripts.

The identification of altered biological pathways as early events in living sporadic PD subjects [61] may direct future investigation in the search of validated drug targets for therapeutic intervention. In this context, it will be interesting to study the role of epigenomic changes in PD and the use of epigenetic modifiers as PD candidate drugs.

\section{Additional files}

\begin{abstract}
Additional file 1: Venn diagram of probe sets scored as present calls following three different amplification methods. Blood collected from a single healthy donor was divided in 3 groups of 2 samples each, and each group was processed for microarray target preparation according to 1) Affymetrix one - cycle cDNA synthesis/ Affymetrix IVT (Affymetrix), 2) Illumina probe synthesis protocol (Ambion), 3) Ovation ${ }^{\text {TM }}$ Whole Blood Solution (NuGEN). Total RNA input amounts were based on manufacturer's recommendations for each method. Hybridizations of targets were performed to HG U133A 2.0 arrays (Affymetrix, Santa Clara, CA). Cell intensity values and probe detection calls were computed using the AffymetrixGeneChip Operating Software (GCOS). This Venn diagram shows the overlap of Affymetrix probe sets with detection call "Present" following the three amplification methods (Affymetrix, Illumina and NuGEN). Data are relative to one of the two replicates and are good representatives of both replicates. 3553 present calls were detected exclusively using the NuGEN (Ovation TM Whole Blood Solution) method in a reproducible fashion. Therefore, despite starting from a much lower RNA amount, the NuGEN protocol detected a significant number of transcripts that were not identified with any of the other methods tested in this study ( $29 \%$ and $37 \%$ more, respectively, than with the Illumina and Affymetrix protocols). (PDF $3122 \mathrm{~kb}$ )
\end{abstract}

Additional file 2: Score plots of the first 6 PCs calculated on the dataset constituted by the 395 variables selected by Ranking-PCA. Control samples are represented as filled circles while pathological samples as void circles. Of the original 60 samples, one (a control sample) did not pass the microarray hybridization quality controls and was excluded from further analyses. All results of bioinformatics analyses shown in this paper refer to this set of 59 samples. (PDF $3122 \mathrm{~kb}$ )

Additional file 3: List of the $\mathbf{3 9 5}$ variables selected by Ranking-PCA to discriminate between PD patients and controls. Order of variables, Affymetrix Probe Set IDs, gene symbols and names are indicated. (PDF $3122 \mathrm{~kb}$ )

Additional file 4: Square of the Mahalanobis distance calculated for each sample from both control and pathological class models. (PDF $3122 \mathrm{~kb}$ )

Additional file 5: Main processes (GOTERM_BP_FAT as classified by DAVID Gene Ontology) altered in whole peripheral blood cells in the 395 selected variables comparing PD patients and controls. GO annotations with at least 3 genes and $P<0.05$ (Fisher exact probability) are presented. Count: number of genes involved in the term; \%: percentage of involved genes/total genes; $P$-Value: modified fisher exact $P$-value, EASE Score; Benjamini: adjusted $P$-value using Benjamini-Hochberg procedure. (PDF $3122 \mathrm{~kb}$ )

Additional file 6: List of differentially expressed genes between PD patients and controls based on PUMA. Ratios are calculated as 
differences in gene expression between samples of PD patients and controls, with the top up-regulated genes first. (PDF $3122 \mathrm{~kb}$ )

Additional file 7: List of differentially expressed genes between PD patients and controls based on Significance Analysis of Microarrays (SAM). Significance Analysis of Microarrays was performed on normalized and filtered microarray data as described in the main text. The SAM test was run using 1000 permutations and a False Discovery Rate (FDR) of $10 \%$. Ratios are calculated as differences in gene expression between samples of PD patients and controls, with the top up-regulated genes first. (PDF $3122 \mathrm{~kb}$ )

Additional file 8: List of differentially expressed genes between PD patients and controls based on Rank Product analysis (RP). Rank Product analysis was performed on normalized and filtered microarray data as described in the main text. The RP test was run using 100 permutations and an FDR of $0.005 \%$ and looking for up- and down-regulated genes separately. Low RP-Values indicate high significance of the results. Genes are shown with the most significant first. (PDF $3122 \mathrm{~kb}$ )

Additional file 9: List of common differentially expressed genes between PD patients and controls based on SAM and PUMA analyses and the $\mathbf{3 9 5}$ selected variables of the Ranking-PCA. Genes are ranked according to the P-values based on PUMA analysis. Order of the 395 variables, Affymetrix Probe Set IDs, gene symbols and names are indicated. Names shown in bold indicate transcripts validated by RT-qPCR (Fig. 2a). (PDF $3122 \mathrm{~kb}$ )

Additional file 10: List of common genes between A9 DA neurons of the SN in the mouse and differentially expressed transcripts in the blood of PD patients according to SAM (a) and PUMA (b). (PDF $3122 \mathrm{~kb}$ )

Additional file 11: Statistically significant gene sets from GSEA analyses. GSEA was run on the normalized, unfiltered microarray dataset as suggested in the tools implementation (http://www.broadinstitute.org/ gsea/ version 2.06), and using the c5 - GO gene sets collection of the Molecular Signatures Database (MSigDB) (http://www.broadinstitute.org/ $\mathrm{gsea} / \mathrm{msigdb} /$ ). The test was performed separately on each of the c5 sub-collections (biological process, molecular function and cellular component), running 1000 permutations and excluding gene sets with fewer than 5 genes or more than 150 (the latter, to retain granularity). Names (Gene Symbol) shown in bold indicate transcripts measured by RT-qPCR (Additional file 12). (PDF $3122 \mathrm{~kb}$ )

Additional file 12: Genes involved in chromatin remodeling and methylation are targeted in PD. Selected samples (12 PD and $12 \mathrm{HC}$ blood samples) previously processed for gene expression profiling were also tested in RT-qPCR assays for several targets (CBX5, HELLS, MECP2, ASF1A, DNMT3A and PRMT1) that were identified by data analyses. Retrotranscription was performed using $1 \mu \mathrm{g}$ of RNA and the iSCRIPT $T^{\mathrm{m}}$ cDNA Synthesis Kit (Bio-Rad) according to the manufacturer's protocol. Real Time qPCR was executed using SYBER-Green PCR Master Mix (Applied Biosystem) and an iCycler IQ Real Time PCR System (Bio-Rad). Sequences of gene specific primers are reported in the table below. Expression of the gene of interest was normalized to $\beta$-actin. The relative expression of each sample was calculated by the formula $2 \exp ^{-\Delta \Delta C t}$ (User Bulletin 2 of the ABI Prism 7700 Sequence Detection System). The amplified products were separated on a $2 \%$ agarose gel and visualized with ethidium bromide staining. (PDF $3122 \mathrm{~kb}$ )

Additional file 13: Selection of reliable reference genes for peripheral blood gene expression analyses. TaqMan ${ }^{\oplus}$ array human endogenous control cards (Applied Biosystems, Foster City, CA, USA) are 384-well microfluidic cards containing 16 human TaqMan Gene Expression Assays. They were used to evaluate the endogenous controls specific for peripheral blood that exhibit minimal differential expression. Peripheral blood samples from 8 PD and 8 HC gender- and age-matched subjects were processed following the manufacturer's instructions (Applied Biosystems, Foster City, CA, USA). The expression stability was determined and compared by two commonly used algorithms (geNorm and NormFinder). By comparing the output of these two methods and by accepting gene expression levels of $\mathrm{qPCR}$ at $\mathrm{Ct}$ values $\leq 29$, we obtained a list of the most stable reference genes in human peripheral blood. See the following Figures reporting output files of the analyses. As the best reference genes, PGK1, UBC and GAPDH were selected according to the following practical considerations: PGK1 is the best reference gene according to geNorm and NormFinder analyses; UBC presents similar stability strength to PGK1 and a different threshold Ct value; GAPDH is one of the most widely used reference genes in peripheral blood expression studies. The gene expression analyses of the first experimental data sets (data not shown) reveled that GAPDH had a higher variability compared to PGK1 and UBC; therefore, we decided to evaluate the relative gene expression by normalizing the data to the geometric mean of PGK1 and UBC. (PDF $3122 \mathrm{~kb}$ )

Additional file 14: List of similar- and anti-similar-to-PD compounds from drug network analysis. Community identifiers, drugs and community enrichment $p$-values resulting from the drug network analysis of genome-wide ranked lists of genes sorted according to their differential expression in PD. Drugs are sorted according to their similarity to PD (a) and according to their "anti-similarity" (b) as explained in the main manuscript. (PDF $3121 \mathrm{~kb})$

\section{Abbreviations}

PD: Parkinson's disease; CNS: central nervous system; SN: Substantia Nigra; DA: dopaminergic; de novo: early stage of the disease; drug-naïve: no specific pharmacological treatment; HC: healthy control; SD: Standard Deviation; MMSE: Mini Mental State Examination; SPECT: Single Photon Emission Computed Tomography; PCA: Principal Component Analysis; PLS-DA: Partial Least Square Discriminant Analysis; R-PCA: Ranking-Principal Component Analysis; LDA: Linear Discriminant Analysis; NER\%: non-error rate; RMA: Robust Multi-Array Average; MeV: Multiexperiment Viewer; SAM: Significance Analysis of Microarrays; RP: Rank Product; PPLR: probability of positive log ratio; FDR: False Discovery Rate; GO: Gene Ontology; GSEA: Gene Set Enrichment Analysis; RT-qPCR: Quantitative real-time PCR; PCs: Principal Components; P: P-value.

\section{Competing interests}

The authors state that there are no conflicts of interests regarding the publication of this article.

\section{Authors' contribution}

$\mathrm{RC}$ designed the experiments, performed the experiments and data analyses, wrote and revised the manuscript; GP, MB, LA, TC and AC performed patients' diagnosis, provided their samples and clinical information; PR, ER, $\mathrm{EM}, \mathrm{Fl}, \mathrm{AC}, \mathrm{DB}$ performed the bioinformatic and statistical analyses of the microarray experiments; DL performed first microarray experiments; SF and CV performed qRT-PCR experiments, ACe and NP provided the frame settings and the financially support to develop the early steps of the project; GP and SG conceived, designed and wrote the paper. SG provided support, direction and supervision of the experiments. RC, PR and SG revised the final version of the manuscript. All authors read and approved the final version of the manuscript.

\section{Acknowledgments}

We express our gratitude to patients, control subjects who participated in this study and to "Associazione Donatori di Sangue", Trieste. We are indebted to all the members of the SG lab for support, to Drs Helena Krmac and Cristina Leonesi for technical help, to Andrea Grimalda, Annalisa Sulli, Monica Sirk for administrative work and to Profs Legname and Persichetti for thought-provoking discussions. This work was initially supported by ITALTBS S.p.A. to develop a webbased application for clinical and genomic data collection (SYMPAR). This study was then supported by SISSA intramural grants, by the Italian Ministry of Education, University and Research (FIRB grant prot.RBAP11FRE9) to SG and GP and by the grant GGP10224 of the Telethon Foundation to SG and GP.

\section{Author details}

'Area of Neuroscience, International School for Advanced Studies (SISSA), via Bonomea 265, 34136 Trieste, Italy. ${ }^{2}$ Department of Medical Sciences, Neurology Unit, University of Trieste, Strada di Fiume 447, 34100 Trieste, Italy. ${ }^{3}$ Department of Environmental and Life Sciences, University of Eastern Piedmont, Viale T. Michel 11, 15121 Alessandria, Italy. ${ }^{4}$ Telethon Institute of Genetics and Medicine (TIGEM), Via P. Castellino 111, Naples 80131, Italy. ${ }^{5}$ ITALTBS S.p.A., AREA Science Park, Padriciano, 99, 34149 Trieste, Italy. ${ }^{6}$ CBM Scrl - Consorzio per il Centro di Biomedicina Molecolare, Area Science Park, S.S.14, km 163.5, Basovizza, 34149 Trieste, Italy. ${ }^{7}$ Department Computer Science \& Systems, School of Engineering, University of Naples "Federico II", via Claudio 21, 80125 Naples, Italy. ${ }^{8}$ Present Address: European Molecular 
Biology Laboratory, European Bioinformatics Institute (EMBL-EBI), CB10 1SD Hinxton, Cambridge, UK.

\section{Received: 25 February 2015 Accepted: 2 October 2015} Published online: 28 October 2015

\section{References}

1. Hornykiewicz O. Basic research on dopamine in Parkinson's disease and the discovery of the nigrostriatal dopamine pathway: the view of an eyewitness. Neurodegener Dis. 2008;5:114-7.

2. Chaudhuri KR, Schapira AHV. Non-motor symptoms of Parkinson's disease: dopaminergic pathophysiology and treatment. Lancet Neurol. 2009:8:464-74.

3. Bas J, Calopa M, Mestre M, Molleví DG, Cutillas B, Ambrosio S, et al. Lymphocyte populations in Parkinson's disease and in rat models of parkinsonism. J Neuroimmunol. 2001:113:146-52.

4. Fan $\mathrm{H}$, Hegde PS. The transcriptome in blood: challenges and solutions for robust expression profiling. Curr Mol Med. 2005;5:3-10.

5. Scherzer CR, Eklund AC, Morse LJ, Liao Z, Locascio JJ, Fefer D, et al. Molecular markers of early Parkinson's disease based on gene expression in blood. Proc Natl Acad Sci U S A. 2007;104:955-60.

6. Kedmi M, Bar-Shira A, Gurevich T, Giladi N, Orr-Urtreger A. Decreased expression of B cell related genes in leukocytes of women with Parkinson's disease. Mol Neurodegener. 2011;6:66

7. Potashkin JA, Santiago JA, Ravina BM, Watts A, Leontovich AA. Biosignatures for Parkinson's disease and atypical parkinsonian disorders patients. PLoS One. 2012;7:e43595.

8. Karlsson MK, Sharma P, Aasly J, Toft M, Skogar O, Sæbø S, et al. Found in transcription: accurate Parkinson's disease classification in peripheral blood. J Park Dis. 2013;3:19-29.

9. Mutez E, Larvor L, Leprêtre F, Mouroux V, Hamalek D, Kerckaert J-P, et al. Transcriptional profile of Parkinson blood mononuclear cells with LRRK2 mutation. Neurobiol Aging. 2011;32:1839-48.

10. Soreq $L$, Israel $Z$, Bergman $H$, Soreq $H$. Advanced microarray analysis highlights modified neuro-immune signalling in nucleated blood cells from Parkinson's disease patients. J Neuroimmunol. 2008;201-201: 227-36.

11. Mutez E, Nkiliza A, Belarbi K, de Broucker A, Vanbesien-Mailliot C, Bleuse S, et al. Involvement of the immune system, endocytosis and EIF2 signaling in both genetically determined and sporadic forms of Parkinson's disease. Neurobiol Dis. 2014;63:165-70.

12. Fahn S, Elton RL, and members of the UPDRS Development Committee. Unified Parkinson's Disease Rating Scale. In: Recent developments in Parkinson's disease, vol. 2. Florham Park (NJ): Macmillan Health Care Information; 1987. p. 153-63.

13. Hoehn MM, Yahr MD. Parkinsonism: onset, progression and mortality. Neurology. 1967;17:427-42.

14. Folstein MF, Folstein SE, McHugh PR. "Mini-mental state". A practical method for grading the cognitive state of patients for the clinician. J Psychiatr Res. 1975;12:189-98.

15. Benamer TS, Patterson J, Grosset DG, Booij J, de Bruin K, van Royen E, et al. Accurate differentiation of parkinsonism and essential tremor using visual assessment of [1231]-FP-CIT SPECT imaging: the [123I]-FP-CIT study group. Mov Disord Off J Mov Disord Soc. 2000;15:503-10.

16. Massart DL, Vanderginste BGM, Buydens LMC, De Jong S, Lewi PJ, SmeyersVerbeke J. Handbook of chemometrics and qualimetrics: part A. Amsterdam: Elsevier; 1988.

17. Tormod N, Harald M. Multivariate Calibration. New York.: Wiley; 1989

18. Marengo E, Robotti E, Bobba M, Gosetti F. The principle of exhaustiveness versus the principle of parsimony: a new approach for the identification of biomarkers from proteomic spot volume datasets based on principal component analysis. Anal Bioanal Chem. 2010;397:25-41.

19. McLachlan GJ. Discriminant Analysis and Statistical Pattern Recognition. John Wiley \& Sons; 2004.

20. Pearson RD, Liu X, Sanguinetti G, Milo M, Lawrence ND, Rattray M. puma: a Bioconductor package for propagating uncertainty in microarray analysis. BMC Bioinformatics. 2009;10:211.

21. Tusher VG, Tibshirani R, Chu G. Significance analysis of microarrays applied to the ionizing radiation response. Proc Natl Acad Sci U S A. 2001;98:5116-21.
22. Breitling $R$, Armengaud $P$, Amtmann A, Herzyk P. Rank products: a simple, yet powerful, new method to detect differentially regulated genes in replicated microarray experiments. FEBS Lett. 2004;573:83-92.

23. Ashburner M, Ball CA, Blake JA, Botstein D, Butler H, Cherry JM, et al. Gene ontology: tool for the unification of biology. The Gene Ontology Consortium. Nat Genet. 2000;25:25-9.

24. Dennis Jr G, Sherman BT, Hosack DA, Yang J, Gao W, Lane HC, et al. DAVID: Database for Annotation, Visualization, and Integrated Discovery. Genome Biol. 2003;4:P3.

25. Subramanian A, Tamayo P, Mootha VK, Mukherjee S, Ebert BL, Gillette MA, et al. Gene set enrichment analysis: a knowledge-based approach for interpreting genome-wide expression profiles. Proc Natl Acad Sci U S A. 2005;102:15545-50.

26. Biagioli M, Pinto M, Cesselli D, Zaninello M, Lazarevic D, Roncaglia P, et al. Unexpected expression of alpha- and beta-globin in mesencephalic dopaminergic neurons and glial cells. Proc Natl Acad Sci U S A. 2009;106: 15454-9.

27. Caiazzo M, Dell'Anno MT, Dvoretskova E, Lazarevic D, Taverna S, Leo D, et al. Direct generation of functional dopaminergic neurons from mouse and human fibroblasts. Nature. 2011;476:224-7.

28. Grison A, Zucchelli S, Urzì A, Zamparo I, Lazarevic D, Pascarella G, et al. Mesencephalic dopaminergic neurons express a repertoire of olfactory receptors and respond to odorant-like molecules. BMC Genomics. 2014;15:729

29. Grünblatt E, Zehetmayer $S$, Jacob CP, Müller T, Jost WH, Riederer P. Pilot study: peripheral biomarkers for diagnosing sporadic Parkinson's disease. J Neural Transm. 2010;117:1387-93.

30. Molochnikov L, Rabey JM, Dobronevsky E, Bonucelli U, Ceravolo R, Frosini D, et al. A molecular signature in blood identifies early Parkinson's disease. Mol Neurodegener. 2012;7:26.

31. Iorio F, Bosotti R, Scacheri E, Belcastro V, Mithbaokar P, Ferriero R, et al. Discovery of drug mode of action and drug repositioning from transcriptional responses. Proc Natl Acad Sci U S A. 2010;107:14621-6.

32. Sharma S, Moon CS, Khogali A, Haidous A, Chabenne A, Ojo C, et al. Biomarkers in Parkinson's disease (recent update). Neurochem Int. 2013;63:201-29.

33. Santiago JA, Potashkin JA. Network-based metaanalysis identifies HNF4A and PTBP1 as longitudinally dynamic biomarkers for Parkinson's disease. Proc Natl Acad Sci U S A. 2015;112:2257-62.

34. Thenganatt MA, Jankovic J. Parkinson disease subtypes. JAMA Neurol. 2014:71:499-504

35. Marth JD, Grewal PK. Mammalian glycosylation in immunity. Nat Rev Immunol. 2008;8:874-87.

36. De Pooter RF, Kee BL. E proteins and the regulation of early lymphocyte development. Immunol Rev. 2010;238:93-109.

37. Yi F, Pereira L, Hoffman JA, Shy BR, Yuen CM, Liu DR, et al. Opposing effects of Tcf3 and Tff1 control Wnt stimulation of embryonic stem cell self-renewal. Nat Cell Biol. 2011;13:762-70.

38. Atlasi Y, Noori R, Gaspar C, Franken P, Sacchetti A, Rafati H, et al. Wnt signaling regulates the lineage differentiation potential of mouse embryonic stem cells through Tcf3 down-regulation. PLoS Genet. 2013;9:e1003424.

39. L'Episcopo F, Tirolo C, Testa N, Caniglia S, Morale MC, Serapide MF, et al. Wnt/ $\beta$-catenin signaling is required to rescue midbrain dopaminergic progenitors and promote neurorepair in ageing mouse model of Parkinson's disease. Stem Cells Dayt Ohio. 2014;32:2147-63.

40. Gadea G, Blangy A. Dock-family exchange factors in cell migration and disease. Eur J Cell Biol. 2014;93:466-77.

41. Yelo E, Bernardo MV, Gimeno L, Alcaraz-García MJ, Majado MJ, Parrado A Dock10, a novel CZH protein selectively induced by interleukin-4 in human B lymphocytes. Mol Immunol. 2008;45:3411-8.

42. Feng Y, Jankovic J, Wu Y-C. Epigenetic mechanisms in Parkinson's disease. J Neurol Sci. 2015;349:3-9.

43. Nicholas AP, Lubin FD, Hallett PJ, Vattem P, Ravenscroft P, Bezard E, et al. Striatal histone modifications in models of levodopa-induced dyskinesia. J Neurochem. 2008;106:486-94.

44. Voutsinas GE, Stavrou EF, Karousos G, Dasoula A, Papachatzopoulou A, Syrrou M, et al. Allelic imbalance of expression and epigenetic regulation within the alpha-synuclein wild-type and p.Ala53Thr alleles in Parkinson disease. Hum Mutat. 2010;31:685-91. 
45. Jowaed A, Schmitt I, Kaut O, Wüllner U. Methylation regulates alphasynuclein expression and is decreased in Parkinson's disease patients brains. J Neurosci Off J Soc Neurosci. 2010;30:6355-9.

46. Kontopoulos E, Parvin JD, Feany MB. Alpha-synuclein acts in the nucleus to inhibit histone acetylation and promote neurotoxicity. Hum Mol Genet. 2006;15:3012-23.

47. Tan Y, Wu L, Zhao Z, Wang Y, Xiao Q, Liu J, et al. Methylation of a-synuclein and leucine-rich repeat kinase 2 in leukocyte DNA of Parkinson's disease patients. Parkinsonism Relat Disord. 2014;20:308-13.

48. Desplats $P$, Spencer B, Coffee E, Patel P, Michael S, Patrick C, et al. Alpha-synuclein sequesters Dnmt1 from the nucleus: a novel mechanism for epigenetic alterations in Lewy body diseases. J Biol Chem. 2011;286:9031-7.

49. Sharma SK, Carlson EC, Ebadi M. Neuroprotective actions of Selegiline in inhibiting 1-methyl, 4-phenyl, pyridinium ion (MPP+)-induced apoptosis in SK-N-SH neurons. J Neurocytol. 2003;32:329-43.

50. Sharma SK. Antioxidants As Potential Therapeutics in Neurodegeneration. 2014:2191-2273, Chapter 97

51. Sharma S. Nanotheranostics in evidence based personalized medicine. Curr Drug Targets. 2014;15:915-30.

52. Kelly TK, De Carvalho DD, Jones PA. Epigenetic modifications as therapeutic targets. Nat Biotechnol. 2010;28:1069-78.

53. Xu Z, Li H, Jin P. Epigenetics-based therapeutics for neurodegenerative disorders. Curr Transl Geriatr Exp Gerontol Rep. 2012;1:229-36.

54. Narayan P, Dragunow M. Pharmacology of epigenetics in brain disorders. $\mathrm{Br}$ J Pharmacol. 2010;159:285-303.

55. Wang $Y$, Wang $X$, Li R, Yang Z-F, Wang Y-Z, Gong X-L, et al. A DNA methyltransferase inhibitor, 5-aza-2'-deoxycytidine, exacerbates neurotoxicity and upregulates Parkinson's disease-related genes in dopaminergic neurons. CNS Neurosci Ther. 2013;19:183-90

56. Hiragami K, Festenstein R. Heterochromatin protein 1: a pervasive controlling influence. Cell Mol Life Sci CMLS. 2005;62:2711-26.

57. Okano M, Bell DW, Haber DA, Li E. DNA methyltransferases Dnmt3a and Dnmt3b are essential for de novo methylation and mammalian development. Cell. 1999:99:247-57.

58. Samaco RC, Neul JL. Complexities of Rett Syndrome and MeCP2. J Neurosci Off J Soc Neurosci. 2011:31:7951-9.

59. Moshkin YM, Armstrong JA, Maeda RK, Tamkun JW, Verrijzer P, Kennison JA, et al. Histone chaperone ASF1 cooperates with the Brahma chromatinremodelling machinery. Genes Dev. 2002;16:2621-6.

60. Liu G, Yu J, Ding J, Xie C, Sun L, Rudenko I, et al. Aldehyde dehydrogenase 1 defines and protects a nigrostriatal dopaminergic neuron subpopulation. J Clin Invest. 2014;124:3032-46.

61. Soreq L, Guffanti A, Salomonis N, Simchovitz A, Israel Z, Bergman H, et al. Long non-coding RNA and alternative splicing modulations in Parkinson'sleukocytes identified by RNA sequencing. PLoS Comput Biol. 2014;10(3):e1003517.

\section{Submit your next manuscript to BioMed Central and take full advantage of:}

- Convenient online submission

- Thorough peer review

- No space constraints or color figure charges

- Immediate publication on acceptance

- Inclusion in PubMed, CAS, Scopus and Google Scholar

- Research which is freely available for redistribution 